\title{
HST imaging of Jupiter shortly after each impact: Plumes and fresh sites
}

\author{
By HEIDI B. HAMMEL \\ Department of Earth, Atmospheric, and Planetary Sciences, Massachusetts Institute of \\ Technology, Cambridge, MA 02139, USA
}

During the first few hours after each impact, numerous phenomena were observed with telescopes on Earth, in orbit, and in space. The primary events in that time were: impacts themselves, rise and fall of large plumes of ejected material, and atmospheric waves; also of interest were the characteristic morphologies of fresh sites. Based on timing from Galileo instruments and groundbased observations, the Hubble Space Telescope (HST) recorded actual impact phenomena for fragments $G$ and $W$, with the $A$ and $E$ impacts occurring just prior to the HST observation window. For these four events, plumes were directly imaged; plume development and collapse correlated with strong infrared emission near the jovian limb, supporting the interpretation that the IR brightness was created by the fall-back of plume material from high altitude (see chapter by Nicholson). For medium-to-large fresh impact sites imaged by HST within a few hours of impact, expanding rings were detected, caused by horizontal propagation of atmospheric waves (see chapters by Ingersoll and Zahnle). Initial site morphology at visible wavelengths was similar for all medium-to-large impacts: a dark streak surrounded by dark material, dominated by a large crescent-shaped ejecta to the southeast. Smaller impact sites typically only showed a dark patch (no ejecta) which dissipated quickly. This chapter summarizes the most recent measurements and interpretations of plumes and fresh impact sites as observed by HST.

\section{Introduction}

Because of its high spatial resolution, the Wide Field and Planetary Camera 2(WFPC2) on Hubble Space Telescope (HST) provided unique views of several phenomena occurring in the initial hours after impact of the Shoemaker-Levy 9 fragments: in particular, the dynamics of plume development were directly imaged, multiple rings were resolved surrounding the moderate and large impact sites, and detailed structure was discernible in the fresh impact sites themselves (Hammel et al. 1995). In the months since the collision, progress has been made both in quantifying these observations, and in interpreting them in the context of the more complete record of ground-based and Galileo data. In this chapter, I review the HST results, present new analyses of some data, and discuss the current interpretation of the observations.

\section{Data overview}

The atmospheric imaging group of the HST Comet Science Team used a total of forty-three orbits for WFPC2 observations of dynamical effects in Jupiter's atmosphere generated by the impact of the comet fragments (Hammel et al. 1995). The team scheduled the majority of the orbits for the week of impacts (195 images), reserving six orbits (75 images) for pre-impact characterization and nine orbits for observations of postimpact evolution (177 images). The team defined a set of filters (see Table 1 of Hammel et al. 1995), and cycled through them as often as possible.

The 0.045-arcsecond pixels of the Planetary Camera (PC) subtended about $171 \mathrm{~km}$ at the sub-Earth point on Jupiter; pixels for Wide Field Camera detector 3 (WF3) were 0.099 arcseconds or roughly $375 \mathrm{~km}$ (the pixel-to- $\mathrm{km}$ ratio varied with both time and position on the planet). Both cameras were used, depending both on the expected 


\begin{tabular}{|c|c|c|c|c|c|}
\hline \multicolumn{2}{|c|}{ Impact $\dagger$} & Frame $\ddagger$ & Filter & $\operatorname{Exp}(\mathrm{s})$ & UT Description" \\
\hline \multirow[t]{6}{*}{ A } & $0<01$ & FQCH4N & 14.0 & $20: 13: 17$ & Emission in shadow \\
\hline & $0 \mathrm{c02}$ & FQCH4N & 4.0 & $20: 15: 17$ & Nothing visible \\
\hline & $0 \mathrm{c03}$ & F953N & 16.0 & 20:18:17 & Plume in sunlight \\
\hline & 0c04 & F547M & 0.16 & $20: 21: 17$ & Plume spreading \\
\hline & $0 \mathrm{c} 05$ & F $410 \mathrm{M}$ & 2.0 & $20: 24: 17$ & Plume falling \\
\hline & $0 \mathrm{c} 06$ & F $336 \mathrm{~W}$ & 3.5 & $20: 27: 17$ & Plume settled to disk \\
\hline \multirow[t]{4}{*}{7} & 0g01 & $\mathrm{FQCH} 4 \mathrm{P} 15$ & 30.0 & $15: 19: 17$ & Plume in sunlight,thermal tail \\
\hline & 0g02 & FQCH4P15 & 16.0 & $15: 21: 17$ & Larger plume \\
\hline & $0 \mathrm{~g} 03$ & F953N & 35.0 & $15: 24: 17$ & Plume falling \\
\hline & 0g04 & F555W & 0.3 & $15: 27: 17$ & Plume settled to disk \\
\hline & $0 \circ 02$ & FQCH4P15 & 30.0 & $07: 33: 17$ & Emission in shadow from meteor \\
\hline & 0003 & FQCH4P15 & 16.0 & $07: 35: 17$ & Emission in shadow \\
\hline & $0 \circ 04$ & F953N & 16.0 & 07:38:17 & Plume in sunlight, thermal tail \\
\hline & $0 \circ 05$ & F555W & 0.3 & $07: 41: 17$ & Larger plume \\
\hline & 0006 & F410M & 10.0 & $07: 44: 17$ & Even larger plume \\
\hline & 0007 & F336W & 18.0 & $07: 51: 17$ & Plume settled to disk \\
\hline \multirow[t]{5}{*}{ W } & 1n05 & F555W & 0.3 & $08: 06: 17$ & Emission in shadow from meteor \\
\hline & $1 \mathrm{n} 06$ & F410M & 10.0 & $08: 09: 17$ & Plume in sunlight \\
\hline & 1n07 & F336W & 18.0 & $08: 16: 17$ & Larger plume, streak at base \\
\hline & 1n 08 & FQCH4P15 & 100.0 & $08: 20: 17$ & Plume settling to disk \\
\hline & $\ln 09$ & FQCH4P15 & 30.0 & $08: 23: 17$ & Plume settled to disk \\
\hline
\end{tabular}

$\dagger$ Images with initial plume phenomena are listed; see Table 3 of Hammel et al. (1995).

$\ddagger$ Frame prefix "u2f" has been omitted.

If Filters differ for A because the observations were made with WF3 rather than PC1.

" Universal time on 1994 dates: A-16 July; E-17 July; G-18 July; W-22 July.

TABLE 1. Selected images from HST plume sequences

phenomena (e.g., plumes or first looks at impact sites) and on circumstances of the HST orbit (sometimes event timing necessitated imaging during the South Atlantic Anomaly, forcing the choice of WF3).

\section{Plumes}

On all four targeted impact events (A, E, G, and W), HST images showed plumes of ejected material above the limb of Jupiter (Fig. 1); see Table 1, adapted from Hammel et al. (1995). The time scale from first detection to final settling into the upper atmosphere of Jupiter ranged from 15 to 20 minutes. Prior to impact, the optical properties of the plume phenomena were highly uncertain; therefore, filters were changed between each exposure in order to maximize the probability of detecting a plume. Plume images later in the impact week were also used to study impact sites created earlier, which required complete filter coverage.

\subsection{Detections of impacts}

In both the $G$ and $W$ plume sequences, bright pixels were seen above the limb of Jupiter in images taken at or very near to the time of impact (Fig. 1, Table 1). The initial G impact signal detected by the Galileo Photopolarimeter Radiometer (PPR) occurred at 7:33:32 UT at $945 \mathrm{~nm}$ (Martin et al. 1995). The Galileo Near-Infrared Mapping 


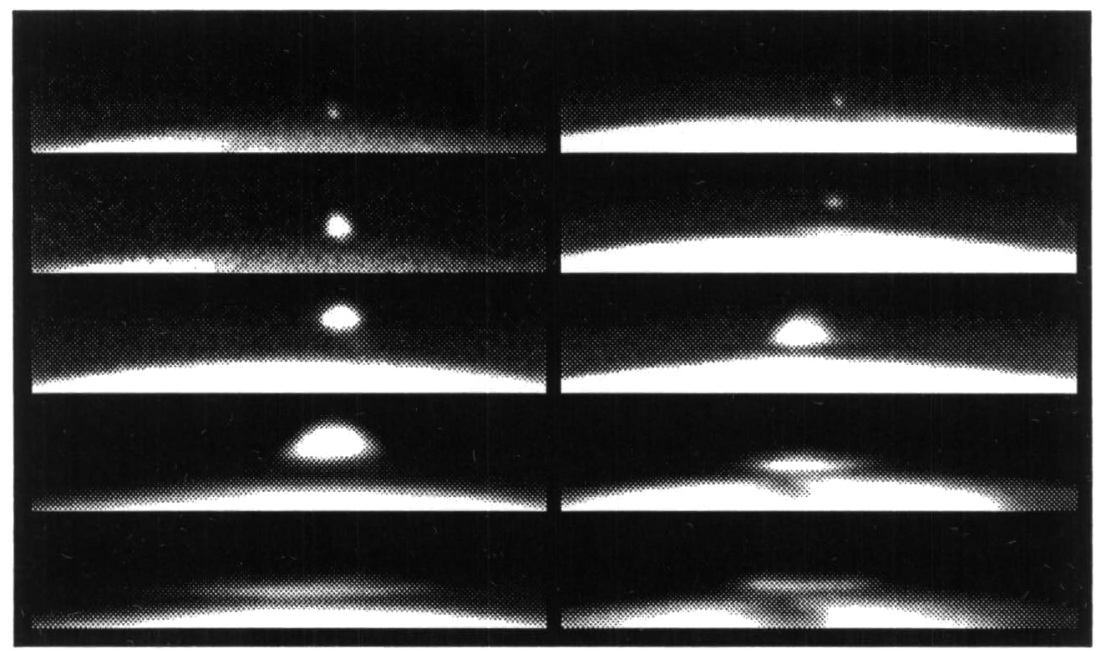

FIGURE 1. Selected plume images from the impacts of fragments $G$ (left) and $W$ (right). The full plume sequences are presented in Hammel et al. (1995); see Table 1 for filters and times. In both columns, the top image was taken at or very near the impact of impact; the subsequent temporal sampling differs in the columns. Both sequences were imaged with the PC. (a) The $\mathrm{G}$ impact created one of the largest impact sites; only $\mathrm{K}$ and $\mathrm{L}$ were bigger. In the third image shown here (taken 5 minutes after impact), bright pixels are seen both in emission in Jupiter's shadow, and in reflection above the shadow. The images are, from top to bottom, frames 0002 , $0003,0004,0006$, and 0o07; time from top to bottom is 15 minutes. (b) The $\mathrm{W}$ impact was the last and hence was closest to the jovian limb. Bright pixels appear to extend northward from the plume in the third image (10 minutes after impact). The images are, from top to bottom, frames $0 \mathrm{n} 05,0 \mathrm{n} 06,0 \mathrm{n} 07,0 \mathrm{n} 08$, and $0 \mathrm{n} 09$; time from top to bottom is 17 minutes.

Spectrometer also detected an event at $7: 33: 37$ at several wavelengths between 0.7 and $5.3 \mu \mathrm{m}$ (Carlson et al. 1995). An 889-nm image taken with HST from 7:33:17 to 7:33:47 UT (encompassing the Galileo first detection) clearly reveals bright pixels in the shadow of Jupiter. PPR data indicate that the initial $\mathrm{G}$ flash lasted for 50 seconds (Martin et al. 1995).

A similar phenomenon was detected in the HST sequence of W plume images. A 555-nm image of the $\mathrm{W}$ impact was taken by HST at 08:06:17. The image, which shows bright pixels in jovian shadow, was obtained within 0.5 second of a $559-\mathrm{nm}$ image from the Galileo Solid-State Imaging (SSI) experiment at 08:06:16.67 (Chapman et al. 1995). The SSI image was second in a sequence of three images (each separated by $21 / 3$ seconds) where emission first appears from the $\mathrm{W}$ impact event. The rapid rise of the SSI signal has been interpreted as radiation emitted by the initial meteor, suggesting a similar interpretation for the initial HST W signal.

It is unlikely that HST detected impact debris rising above the limb in these first G and $\mathrm{W}$ plume images. The $\mathrm{G}$ impact occurred beyond the horizon as seen from HST; thus material would have to have been $444 \mathrm{~km}$ above the $100-\mathrm{mbar}$ limb to be visible (atmospheric refraction is not a significant effect, reducing the distance by only $45 \mathrm{~km}$ ). Similarly, for the $W$ impact, material would have to be $137 \mathrm{~km}$ above the $100-\mathrm{mbar}$ surface to be visible from Earth (refraction reduces this distance by less than $25 \mathrm{~km}$ ). In both cases, to be visible to HST so shortly after impact, ejected material would have to rise with a velocity higher than was inferred from later plume images (Hammel et al. 1995).

These images may have captured the incoming meteors for $\mathrm{G}$ and $\mathrm{W}$. Alternatively, they may have detected light from the meteor scattering off infalling cometary dust or 
meteor light reflected off dust left in the atmosphere by the break-up of the fragment upon entry. It may even be a combination of these effects; further analysis has still not yet yielded conclusive answers for the source of these bright pixels. The chapter by Nicholson has further discussion of these initial events, placing them in the context of the many excellent ground-based lightcurves.

Hammel et al. (1995) had suggested that the image at 20:13:17 UT on 16 July 1994, which showed bright pixels in the vicinity of the A impact site, may have been a detection of the impact itself. However, subsequent verification of the timing from groundbased near-infrared observations at Calar Alto indicated the impact probably occurred at 20:11:59 \pm 5 seconds (Herbst et al. 1995). Thus, the 20:13:17 image probably captured the subsequent rising plume in jovian shadow, as was seen for the $G$ and $W$ plumes (Hammel et al. 1995). Although nothing was detected in the next HST image at 20:15:17, that exposure time was much shorter than that of the previous image (at the same wavelength) and it is also probable that the plume was cooling (i.e., fading) quickly. The third image in the sequence clearly shows the plume in sunlight (Hammel et al. 1995).

\subsection{Plume geometry}

Initial calculations of plume heights as a function of time for the A, E, G, and W plumes suggested that all observed plumes attained nearly identical terminal altitudes near $3000 \mathrm{~km}$ within 6-8 minutes of impact, and were falling and spreading within $10 \mathrm{~min}$ utes of impact (Hammel et al. 1995). Identical plume heights for explosions of different energies were not predicted (Ahrens et al. 1994a, Boslough et al. 1994a, Boslough et al. 1994b, Zahnle and Mac Low 1994).

The team has since refined the plume height measurements with more rigorous image navigation (determination of the planet's location) and added measurements of the widths of the plumes at their base as defined by the shadow of Jupiter (Fig. 2). Note that the plume widths must be used with care, since the altitude of the shadow of Jupiter with respect to the 100-mbar level varies both with differing geometry for different impacts and with time for any single impact.

The more rigorous measurements have confirmed the earlier findings, and provided more details for models. The $G$ impact seems to have remained elevated longer and then collapsed faster than the other three observed plumes (Fig. 2). Sophisticated modeling may indicate whether this is an intrinsic property of a larger plume, or whether differing viewing geometry may be involved.

Hammel et al. (1995) pointed out that the seventh image of the $W$ sequence showed an unexplained feature: material extended northward beyond the edge of the well-defined plume top (Fig. 1). They suggested that this may be debris "splashing" outward, as predicted in some pre-impact models (Mac Low and Zahnle 1994), and may be seen only in the $\mathrm{W}$ impact because of its position (it hit closest to the Jovian limb). However, because the $\mathrm{W}$ impact occurred on the site of the $\mathrm{K}$ impact (one of the largest impacts), there was also speculation that the material may have been residual elevated debris from the $\mathrm{K}$ impact. The team has subsequently checked other images where large sites ( $\mathrm{G}$ and L) appear on the limb, searching for elevated debris. None has been seen, strengthening the suggestion that this material is related to the $\mathrm{W}$ impact itself.

\section{Fresh Sites}

\subsection{Timing based on site location}

Fifteen fragments left clear evidence of impact sites (Table 2); sites for smaller fragments ( F, P2, T, U, and V, along with "missing" fragments $\mathrm{J}$ and $\mathrm{M}$ ) were initially uniden- 


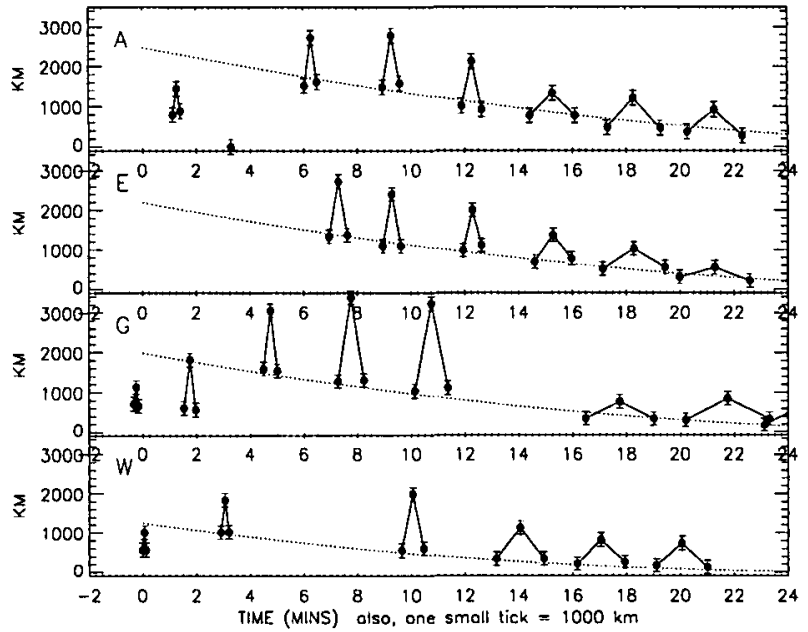

Figure 2. Plume height and width as a function of time. In each panel, the $\mathbf{x}$ axis indicates both time from impact (large ticks) and plume width (small ticks); the y axis indicates height above the 100-mbar level. For each image, the three points indicate the maximum height of the plume and the width of the plume measured at its apparent base, that is, where it rises above Jupiter's shadow into reflected sunlight (when the plume is in shadow, the width is the broadest part of the plume). The dashed lines indicate the calculated height of the jovian shadow above the 100-mbar level (P. Chodas, personal communication). Assumed impact times in this figure were $\mathrm{A}=20: 12: 00, \mathrm{E}=15: 12: 00, \mathrm{G}=7: 33: 32$, and $\mathrm{W}=8: 06: 14$ (all UT).

tified (Hammel et al. 1995). With more accurate timing from ground-based infrared lightcurves, it is probable that sites for several of these latter fragments will be recovered (e.g., F, P2); this work is in progress. Table 2 gives latitudes and longitudes of the detected sites (the positions of the fresh impact sites and, if a ring was observed, the position of the ring's origin).

Impact times (Table 2) were inferred from the difference between predicted and observed longitudes. The HST times should be use with caution: subsequent comparisons with impact times inferred from ground-based and Galileo observations have shown a tendency for the HST times inferred from sites to be on average 2.5 minutes late, ranging from 10 seconds early for fragment $\mathrm{D}$ to more than 6 minutes late for fragment $\mathrm{L}$ (likely due to difficulty in defining a precise "site" for the large, complicated L impact scar).

\subsection{Site sizes}

Hammel et al. (1995) classified each impact site by its apparent size in the first available image after impact (Table 2); subsequent analysis has not significantly changed those results. Nicholson independently assessed the "size" of each impact based on its peak IR flux, finding a general correlation with the HST image classifications (see chapter by Nicholson). Similarly, comparisons of peak Galileo PPR signal values for several impacts agreed with the HST and ground-based IR assessments (Martin et al. 1995). Although these classes agree roughly with pre-impact fragment brightnesses (Weaver et al. 1995), there is some discrepancy: for example, see Figs. 1 and 2 of A'Hearn et al. (1995). The scatter in the correlation may suggest inhomogeneity in fragment strength or perhaps composition (though the latter is less likely). 


\begin{tabular}{|c|c|c|c|c|c|c|}
\hline $\operatorname{Impact}^{\dagger}$ & Class $\ddagger$ & Method & Lat. $\rrbracket$ & Long. & Frame & Inferred Time (UT) \\
\hline \multirow[t]{2}{*}{$A=21$} & $2 \mathrm{a}$ & Ring & $-43.41 \pm 0.05$ & $187.8 \pm 0.3$ & - & $20: 15: 54 \pm 1 \mathrm{~min}$ \\
\hline & & Site & $-43.54 \pm 1.0$ & $186.3 \pm 2.0$ & $0 \mathrm{i} 04$ & $20: 13: 24 \pm 3 \mathrm{~min}$ \\
\hline$B=20$ & 3 & Site & $-42.79 \pm 1.0$ & $71.1 \pm 2.0$ & og05 & $02: 56: 09 \pm 3 \mathrm{~min}$ \\
\hline$C=19$ & $2 \mathrm{a}$ & Site & $-43.41 \pm 1.0$ & $225.0 \pm 2.0$ & $0 \mathrm{i} 04$ & $07: 13: 51 \pm 3 \mathrm{~min}$ \\
\hline$D=18$ & 3 & Site & $-43.29 \pm 1.0$ & $33.5 \pm 2.0$ & og05 & $11: 52: 50 \pm 3 \mathrm{~min}$ \\
\hline \multirow[t]{2}{*}{$E=17$} & $2 a$ & Ring & $-43.48 \pm 0.05$ & $153.5 \pm 0.2$ & - & $15: 12: 11 \pm 1 \mathrm{~min}$ \\
\hline & & Site & $-44.54 \pm 1.0$ & $153.5 \pm 2.0$ & 0i04 & $15: 12: 11 \pm 3 \mathrm{~min}$ \\
\hline \multirow[t]{3}{*}{$\mathrm{G}=15$} & 1 & Ring & $-43.65 \pm 0.04$ & $25.7 \pm 0.2$ & 一 & $07: 33: 17 \pm 1 \mathrm{~min}$ \\
\hline & & Site & $-43.66 \pm 1.0$ & $26.8 \pm 2.0$ & 0p03 & $07: 35: 11 \pm 3 \mathrm{~min}$ \\
\hline & & Plume & - & - & $0 \circ 02$ & $07: 33: 16 \pm 0.5 \mathrm{~min}$ \\
\hline $\mathrm{H}=14$ & $2 a$ & Site & $-43.66 \pm 1.0$ & $101.4 \pm 2.0$ & 0v03 & $19: 33: 21 \pm 3 \mathrm{~min}$ \\
\hline$K=$ & 1 & Site & $-43.29 \pm 1.0$ & $282.6 \pm 2.0$ & 1905 & $10: 30: 58 \pm 3 \mathrm{~min}$ \\
\hline 11 & 1 & Site & $-42.79 \pm 1.0$ & $351.6 \pm 2.0$ & 6803 & $22: 21: 44 \pm 3 \mathrm{~min}$ \\
\hline $\mathbf{N}=$ & 3 & Site & $-43.41 \pm 1.0$ & $73.1 \pm 2.0$ & $1 \mathrm{a} 03$ & $10: 30: 09 \pm 3 \mathrm{~min}$ \\
\hline $\mathrm{Q} 2=7 \mathrm{~b}$ & 3 & Site & $-44.67 \pm 1.0$ & $47.5 \pm 2.0$ & $1 \mathrm{a} 03$ & $19: 46: 31 \pm 3 \mathrm{~min}$ \\
\hline \multirow[t]{2}{*}{$\mathrm{Q} 1=\mathbf{7 a}$} & $2 b$ & Ring & $-44.37 \pm 0.1$ & $64.0 \pm 0.5$ & - & $20: 14: 42 \pm 1 \mathrm{~min}$ \\
\hline & & Site & $-43.41 \pm 1.0$ & $66.3 \pm 2.0$ & $1 \mathrm{a} 03$ & $20: 18: 24 \pm 3 \mathrm{~min}$ \\
\hline \multirow[t]{2}{*}{$R=6$} & $2 b$ & Ring & $-44.17 \pm 0.1$ & $46.8 \pm 0.5$ & & $05: 41: 18 \pm 1 \mathrm{~min}$ \\
\hline & & Site & $-44.50 \pm 1.0$ & $43.6 \pm 2.0$ & $1 \mathrm{a} 03$ & $05: 36: 06 \pm 3 \mathrm{~min}$ \\
\hline$S=5$ & $2 c$ & Site & $-43.91 \pm 1.0$ & $34.0 \pm 2.0$ & 1106 & $15: 17: 46 \pm 3 \mathrm{~min}$ \\
\hline \multirow[t]{2}{*}{$\mathrm{W}=1$} & $2 \mathrm{c}$ & Site & $-44.29 \pm 1.0$ & $284.8 \pm 2.0$ & 1003 & $08: 08: 46 \pm 3 \mathrm{~min}$ \\
\hline & & Plume & 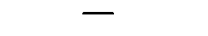 & - & $\ln 05$ & $08: 06: 16 \pm 0.1 \mathrm{~min}$ \\
\hline
\end{tabular}

$\dagger$ Impact sites for $F=16, P 2=8 b, T=4, U=3$, and $V=2$ were not detected. $J=13, M=10$, and $P 1=8 \mathrm{a}$ are omitted because they faded from view (the letters $I$ and $O$ were not used).

$\ddagger$ Impact site size based on first view after impact. Class $1=$ dark region $>10000 \mathrm{~km}$, large ejecta, probably multiple rings; Class $2 a=4000<r<8000 \mathrm{~km}$, medium ejecta, possibly multiple rings; Class $2 b=$ medium but slightly smaller ejecta, probably single ring; Class $2 c(S$, $W)=<6000 \mathrm{~km}$, classification based on ground-based data, impacts occurred on earlier sites; Class $3=<3000 \mathrm{~km}$, no ejecta, no ring; Class $4=$ not detected.

I Method of determining site location and time; multiple images were used for positions based on ring measurements. Latitudes are planetocentric; longitudes are System III. "Frame" is the image used to measure latitude and longitude of impact site (the prefix " $u 2 f$ " has been deleted).

"To determine impact times for "sites" and "rings," the time was obtained by finding the difference in longitude between HST measurements and predictions, and assuming a rotation period of $9.92492 \mathrm{hrs}$ (1.654153 mins/deg); for "plumes," the time was that of first image showing brightening.

TABLE 2. Relative sizes, locations, and times of impacts

\subsection{Fresh site morphology}

The medium-to-large fresh impact sites showed a consistent morphology (Hammel et al. 1995): a large crescent-shaped ejecta offset toward the southeast from a streak, with fainter traces of dark material extending all around the impact site (Fig. 3). The largest sites showed a sharp circular ring and sometimes a second faint inner ring (concentric with the main ring but visible mainly northwest of the ring center). The ring center presumably marks the point of maximum energy release. When a ring was seen, the streak extended from the ring center to the southeast toward the ejecta.

The crescent shape of the ejecta suggests that the range of elevation angles is small, although this is still under discussion (K. Jessup, personal communication; also see chapter by Crawford). The material is not highly collimated in azimuth: the crescent extends 


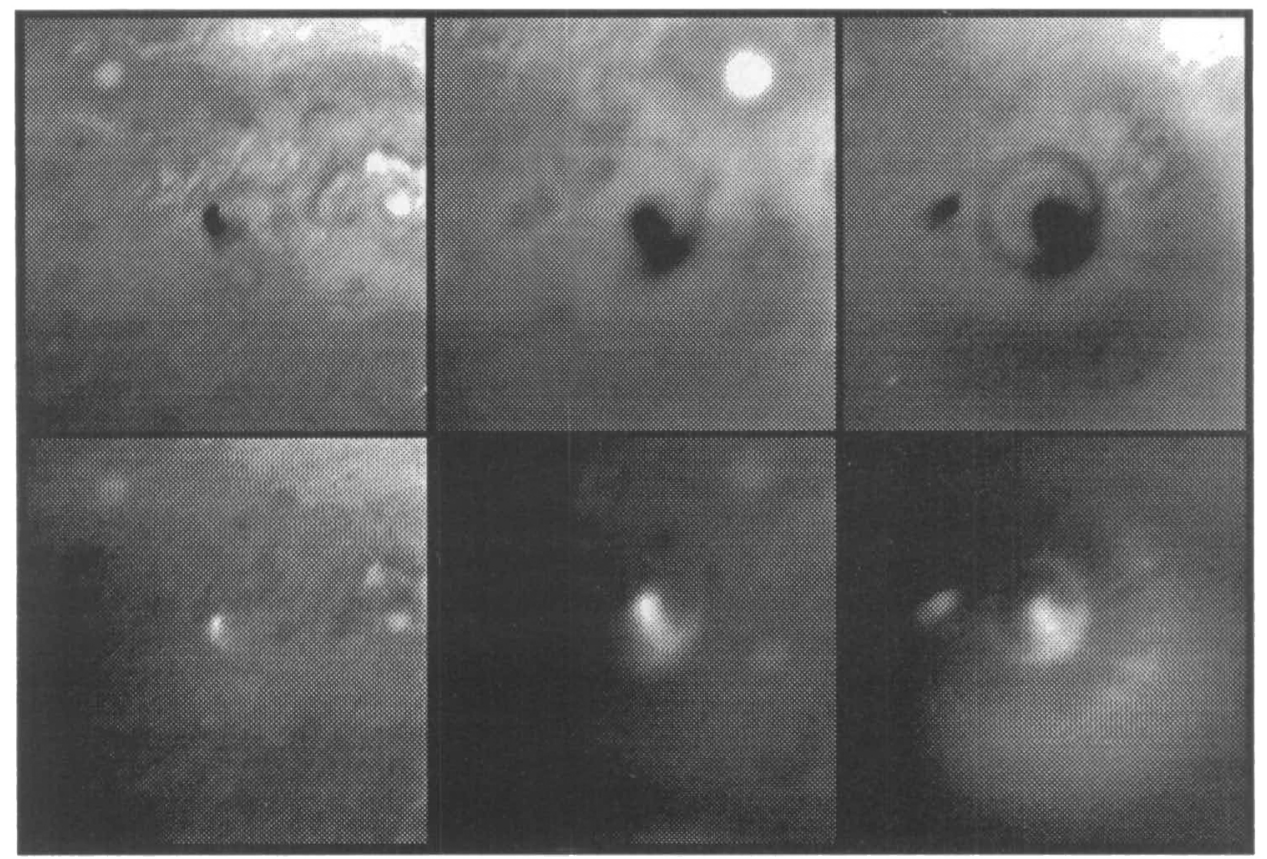

Figure 3. Fresh sites. These images show three fresh impact sites, ranging in size from small (D, left images, 2.03 hours after impact) to medium ( $E$, middle images, 1.86 hours after impact) to large ( $G$, right images, 1.66 hours after impact; the small site to the left of $G$ is the $D$ site about 10 hrs later). Upper $D$ and $E$ images are at $410 \mathrm{~nm}$, the upper $G$ image in at $555 \mathrm{~nm}$, and all bottom row images are at $889 \mathrm{~nm}$. The images of D (frames $0 \mathrm{f} 01$ and $0 \mathrm{f} 05$ ) and $\mathrm{G}(0 \mathrm{p} 01$ and 0 p04) were made with the PC; the E images (0h01 and 0h05) were taken with WF3.

at least $180^{\circ}$ around the impact site. As discussed in Hammel et al. (1995), the size of the $\mathrm{G}$ ejecta implies ejection velocities of more than $10 \mathrm{~km} / \mathrm{sec}$ (see below), which is consistent with the observed plume heights.

Rays (linear features) were seen in the crescent of the $G$ impact (no images were obtained soon enough after the $\mathrm{K}$ and $\mathrm{L}$ impacts to search for rays in their ejecta); the rays seemed to emanate from a point slightly $(1000-2000 \mathrm{~km})$ to the southeast of the ring center, near the end of the streak (Fig. 3). The apparent emanation point of the rays may refer to the center of collapse of the $G$ plume, which is offset from the base of the plume. If the plume material was uniformly ejected, the rays may be indicative of inhomogeneities in the jovian atmosphere into which the plume collapsed. Alternatively, the linear features could instead be arcs of material that were thrown out by irregular events during the ejection process itself. The precise nature of these features is still unknown.

Smaller impact sites showed only the central streaks, i.e., there was little obvious ejecta to the southeast (Fig. 3). However, the detection of IR "main events" for some of these smaller sites suggests that material was ejected (see chapter by Nicholson); presumably it was too optically thin to be detected in HST images.

\subsection{Site Color}

In the 889-nm methane absorption band, impact debris was brighter than the normal jovian clouds (Fig. 3), suggesting that it was at relatively high altitude, above most of the methane gas. At other wavelengths, including the $619-\mathrm{nm}$ methane band, the impact debris appeared darker than the normal jovian clouds. The color of the ring material 
appeared to match that of the streak and ejecta blanket, suggesting similar composition, but that gave little insight into whether the material was cometary, jovian, or a mixture. One possibility may be that the material is primarily jovian gas with a small amount of cometary debris that has been heated to temperatures of $10,000 \mathrm{~K}$ or more (Martin et al. 1995), and has subsequently cooled, recombining to form complex hydrocarbons enhanced with residual non-organic cometary material. West et al. (1995) point out that the color of the material is consistent with organic material rich in sulfur and nitrogen. In addition, Noll et al. (1995) discuss chemistry of the features derived from HST spectra, which give more information than color alone. For more discussion about the nature of the dark material, see chapters by Lellouch, Moses, and West.

\subsection{Angle of Ejecta}

The azimuth of the ejecta's symmetry axis is determined by both the fragment's initial entry angle, and the subsequent flight of the ejected material through Jupiter's rotating atmosphere. Fragments entered the atmosphere at an elevation angle of $45^{\circ}$, with azimuth angle $16^{\circ}$ counterclockwise from south in a reference frame rotating with Jupiter. Models of oblique impacts (Ahrens et al. 1994b, Boslough et al. 1994a) predicted initial ejection of material back along this same trajectory. Based on the $\mathrm{G}$ impact ejecta's horizontal extent of $13000 \mathrm{~km}$ and an assumed $45^{\circ}$ exit angle for ballistic particles, the ejection velocity of that impact was estimated to be on the order of $17 \mathrm{~km} \mathrm{sec}^{-1}$. This would correspond to a total flight time of about 17 minutes (Hammel et al. 1995). Planetary rotation during that time would add an additional azimuth angle of about $7^{\circ}$ (calculated as $\Omega t \sin \lambda$, where $\Omega$ is the angular velocity of the planet, $t$ is the time, and $\lambda$ is the planetocentric latitude of the impact site), giving a total azimuth angle from south of $23^{\circ}$. The observed azimuth (Fig. 3 ), $35^{\circ} \pm 5^{\circ}$, appears to indicate that the material was "in flight" for 45 minutes, although images show the plumes have almost fully collapsed within about half that time (Fig. 1; Table 1). One explanation is that ejected material slid or bounced along the top of the atmosphere following reentry; if friction with the underlying layer was low, the azimuthal rotation would have been the same as if the material followed a single ballistic trajectory for $\mathbf{4 5}$ minutes.

\section{Waves}

Images taken within a few hours of the larger impacts revealed expanding sharply defined "rings" that were almost certainly caused by atmospheric waves of some sort. The most dramatic (and hence most often shown) example was the multiple ring system created by the large $\mathrm{G}$ fragment (Fig. 3), although rings were also seen after the A, E, R, and Q1 impacts (Hammel et al. 1995).

These rings appear to expand with a speed independent of impact energy. Hammel et al. (1995) measured the radii of the circular features using several techniques. Figure 4 shows positions of the main rings seen after the A, E, G, Q1, and R impacts, as well as those of the inner rings from $\mathrm{E}$ and $\mathrm{G}$.

For the main rings, a best-fit line yielded a slope (i.e., propagation velocity) of $454 \pm$ $20 \mathrm{~m} \mathrm{sec}^{-1}$ and an initial radius of $586 \pm 125 \mathrm{~km}$. A positive initial radius could arise from either nonlinear (faster) initial propagation or a finite source size. The inner rings' velocity was not well determined by the data, but was probably in the range $180-350 \mathrm{~m} \mathrm{sec}^{-1}$. Assuming inner and outer rings started at the same time and radius (i.e., fixing the intercept at $586 \mathrm{~km}$ ), Hammel et al. (1995) found a velocity of $189 \pm 10 \mathrm{~m} \mathrm{sec}^{-1}$ (formal error), roughly consistent with models suggesting a 3:1 ratio for the speeds of the two fastest modes of a linear tropospheric wave (Ingersoll and Kanamori 1995). 


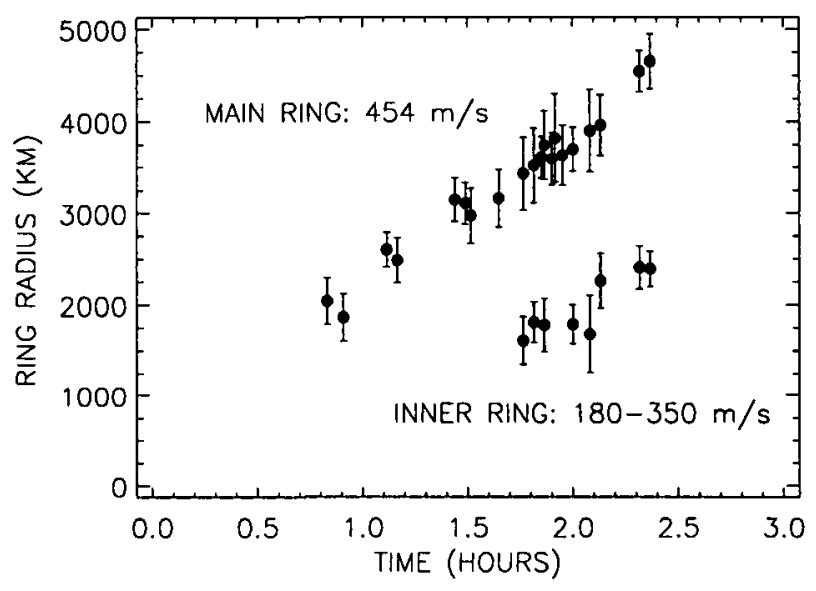

Figure 4. Wave propagation. Each datum is a measurement of the radius of the ring as a function of time from impact for images obtained after five impacts with different explosion energies (A, E, G, Q1, and R). The measurements for the main ring (upper cluster) fall on a straight line, indicating a speed independent of explosion energy. The slope of the inner ring seen in the $\mathrm{E}$ and $\mathrm{G}$ impacts (lower cluster) is less well constrained. See chapters by Ingersoll and Zahnle for discussions of the physical interpretation of the rings.

For a detailed discussion of the linear wave interpretation, see the chapter by Ingersoll. Alternatively, these rings may be a manifestation of nonlinear (breaking) waves, as discussed in the chapter by Zahnle.

\section{Conclusion}

The wealth of detail observed in HST images continues to yield insights into the phenomena resulting from the impact of the fragments of Shoemaker-Levy 9 . The bulk of the burden of unraveling remaining puzzles now lies on modelers, who must not only create models that can reproduce the basic physics of a catastrophic explosion, but must also be able to do this successfully for a large number of events with subtly different initial conditions. Fortunately, the Hubble Space Telescope, along with many ground-based and space-based instruments, has provided a remarkable body of data to fuel these efforts.

HBH acknowledges the expertise and assistance of many people involved with the HST Comet Crash investigation, especially R. Beebe, A. Ingersoll, R. West, and G. Orton, along with J. Mills, A. Simon, T. Dowling, J. Harrington, R. Yelle, and others. Special acknowledgments go to HST Comet Science Team colleagues J. Clarke, H. Weaver, M. McGrath, and K. Noll for their help before, during, and after the crash. These observations were made with the NASA/ESA Hubble Space Telescope, with support provided through grant number GO-5624.08-93A from the Space Telescope Science Institute, which is operated by Association of Universities for Research in Astronomy, Inc., under NASA contract NAS5-26555.

\section{REFERENCES}

A'Hearn, M. F., Meier, R., Wellnitz, D., Woodney, L., Martin, R., Smith, T. \& VERVEER, A. 1995 Were any impact flashes seen in reflection from the satellites? In 
Proceedings of the European SL-9/Jupiter Workshop (eds. R. West and H. Boehnhardt), pp. 113-118. European Southern Observatory.

Ahrens, T. J., Takata, T., O'Keefe, J. D. \& Orton, G. S. 1994a Impact of Comet Shoemaker-Levy 9 on Jupiter. Geophysical Research Letters 21, 1087-1090.

Ahrens, T. J., Takata, T., O'Keefe, J. D. \& Orton, G. S. 1994b Radiative signatures from impact of comet Shoemaker-Levy 9 on Jupiter. Geophysical Research Letters 21, 1551-1553.

Boslough, M. B., Crawford, D. A., Robinson, A. C. \& Trucano, T. G. 1994a Mass and penetration depth of Shoemaker-Levy 9 fragments from time-resolved photometry. Geophysical Research Letters 21, 1555-1558.

Boslough, M. B., Crawford, D. A., Robinson, A. C. \& Trucano, T. G. 1994b Watching for fireballs on Jupiter. EOS 75, 305-310.

Carlson, R. W., Weissman, P. R., Segura, M., Hui, J., Smythe, W. D., Johnson, T. V., Baines, K. H., Drossart, P., Encrenaz, T. \& Leader, F. E. 1995 Galileo infrared observations of the Shoemaker-Levy $9 \mathrm{G}$ impact fireball: a preliminary report. Geophysical Research Letters 22, 1557-1560.

Chapman, C., Merline, W. J., Klaasen, K., Johnson, T. V., Heffernan, C., Belton, M. J. S., Ingersoll, A. P. \& the Galmeo Imaging Team 1995 Preliminary results of Galileo direct imaging of S-L 9 impacts. Geophysical Research Letters 22, 1561-1564.

Hammel, H. B., Beebe, R. F., Ingersoll, A. P., Orton, G. S., Mills, J. R., Simon, A. A., Chodas, P., Clarke, J. T., De Jong, E., Dowling, T. E., Harrington, J., Huber, L. E., KarkoschKa, E., Santori, C. M., Toigo, A., Yeomans, D. \& West, R. A. 1995 Hubble Space Telescope imaging of Jupiter: atmospheric phenomena created by the impact of comet Shoemaker-Levy 9. Science 267, 1288-1296.

Herbst, T., Hamilton, D. P., Bohnhardt, H. \& Ortiz-Moreno, J. L. 1995 Near-infrared imaging and spectroscopy of the SL-9 impacts from Calar Alto. Geophysical Review Letters, submitted.

Ingersoll, A. P. \& Kanamori, H. 1995 Waves from the collisions of comet Shoemaker-Levy 9 with Jupiter. Nature 374, 706-708.

MaC Low, M.-M. \& ZaHNLE, K. 1994 The impact of Shoemaker-Levy 9 on Jupiter. Bull. Amer. Astron. Soc. 26, 926.

Martin, T. Z., Orton, G. S., Travis, L. D., Tamppari, L. K. \& Claypool, I. 1995 Observation of Shoemaker-Levy 9 impacts by the Galileo Photopolarimeter Radiometer. Science 268, 1875-1879.

Noll, K. S., McGrath, M. A., Trafton, L. M., Atreya, S. K., Caldwell, J. J., Weaver, H. A., Yelle, R. V., BARNET, C. \& EDGINGTON, S. 1995 HST spectroscopic observations of Jupiter after the collision of comet Shoemaker-Levy 9. Science 267, 1307-1313.

Weaver, H. A., A'Hearn, M. F., Arpigny, C., Boice, D. C., Feldman, P. D., Larson, S. M., Lamy, P., Levy, D. H., Marsden, B. G., Meech, K. J., Noll, K. S., Scotti, J. V., Sekanina, Z., Shoemaker, C. S., Shoemaker, E. M., Smith, T. E., Stern, S. A., Storrs, A. D., Trauger, J. T., Yeomans, D. K. \& Zellner, B. 1995 The Hubble Space Telescope (HST) observing campaign on comet Shoemaker-Levy 9. Science 267, 1282-1288.

West, R. A., Karkoschka, E., Friedson, A. J., Seymour, M., Baines, K. H. \& Hammel, H. B. 1995 Impact debris particles in Jupiter's stratosphere. Science 267, 1296-1301.

ZAHNLE, K. \& MAC Low, M.-M. 1994 The collision of Jupiter and comet Shoemaker-Levy 9. Icarus 108, 1-17. 